\title{
The grading of cognitive state comparisons with different distances across three conditions in stroke survivors
}

\author{
Yumi Kimª, Yuhyung Park \\ ${ }^{a}$ Department of Physical Therapy, Kangwon National University, Samcheok, Republic of Korea \\ ${ }^{b}$ Department of Physical Therapy, Rehabilitation Medicine Center, Bobath Memorial Hospital, Seongnam, Republic of Korea
}

Objective: The purpose of this study was to compare with different distance across three conditions in stroke survivors with the grading of cognitive state.

Design: Cross-sectional study.

Methods: Twelve stroke patients who agreed to active participation were included. Participants were allotted to normal cognitive $(\mathrm{CN})$ group $(\mathrm{n}=7)$ and cognitive impairment $(\mathrm{CI})$ group $(\mathrm{n}=5)$ and then walked on a self-paced walkway at three conditions on the Time Up and Go (TUG) test and the 6 minute walk test (6MWT): 1) walking with your comfortable speed, 2) walking while carrying a tray with glasses, 3) walking with a verbal cognitive task. The TUG test was repeated three successful times on each condition. For the 6MWT, participants were tested one time.

Results: The CI group walked slower than the CN group at the three conditions on the TUG test. However, there was no significant difference between two groups to each condition. A significant effect of dual tasking was found only in error of verbal cognitive task condition for the TUG test $(p<0.05)$. On the 6MWT, the participants in the CI group walked short distance rather than the $\mathrm{CN}$ group $(p<0.05)$. There were significant differences between two groups not only at all conditions but also at error of verbal cognitive task condition as well $(p<0.05)$.

Conclusions: To consider the results of different distances such as the TUG test and the 6MWT, we think that exercises in long distance would be more effective to patients with CI. Those would be improved patient's endurance in cognitive problem.

Key Words: Cognition, Dual task, Gait, Stroke

\section{Introduction}

Walking is simplistic but has been essentially one of automatic motor tasks. For those tasks, cognitive function is a chief function of communicated walking [1,2]. People need to perform motor tasks and cognitive functions under normal circumstance such as carrying out two different tasks at the same time. Therefore, research about interaction between cognition and motor control has become a focal field to understand recovery of motor control in patients with neurological injury such as stroke.

Dual task study has been used to investigate interactions between cognitive performance and motor behavior [3].
People with neurological deficits such as stroke have been debilitated to gait ability and cognitive performance with dual-task. Several studies about the change of gait or balance during doing a cognitive task have been reported [4,5]. Gait speed and stride length in people with post stroke were decreased while walking on the surface of the ground with dual task [6,7]. Canning et al. [8] also found that walking performance in survivors of stroke can deteriorate (reduced gait speed, stride length, step length, and cadence) under dualand triple-task conditions, similar to that observed in elderly people.

Bowen et al. [9] reported that performing a cognitive task while walking adversely affected stroke patients' balance

Received: 20 August, 2014 Revised: 30 September, 2014 Accepted: 31 October, 2014

Corresponding author: Yuhyung Park

Rehabilitation Medicine Center, Bobath Memorial Hospital, 155-7 Daewangpangyo-ro, Bundang-gu, Seongnam 463-869, Republic of Korea Tel: 82-31-786-3250 Fax: 82-31-786-3026 E-mail: pyh0627@hanmail.net

(c) This is an Open-Access article distributed under the terms of the Creative Commons Attribution Non-Commercial License (http://creativecommons.org/licens es/by-nc/3.0) which permits unrestricted non-commercial use, distribution, and reproduction in any medium, provided the original work is properly cited.

Copyright @ 2014 Korean Academy of Physical Therapy Rehabilitation Science 
and gait velocity. Cockburn et al. [10] also found positive evidence of dual-task gait decrements when a cognitive task was performed concurrently in subjects after stroke. Each patient has different level of dual task interference so the evaluation of a patient's performance about dual task should be considered to treatment process.

Cognitive impairment (CI), acutely or sub acutely, after an acute neurologic event with a stepwise progression is a typical history suggestive of vascular dementia [11]. CI may lead decrease in functional capacity. Therefore, it affects rehabilitation outcomes in stroke [12]. Stephens et al. [13] reported that mild global CI and mild attention loss had negative effect on ADL in the older stroke survivors.

Stroke may cause CI, and the domains most likely to be defective are memory, orientation, language, and attention [14]. Community ambulation is a valuable follow-up measure because of decreased physical capacity and impairment in cognitive functions may interfere with walking ability after stroke [15].

Previous research has not determined which aspects of cognitive activity cause disruption to gait after stroke. Most gait-related dual task studies have examined the effects of only one cognitive task $[9,16]$. Plummer-D'Amato et al. [3] examined the interactions between gait and three different cognitive tasks in a community-dwelling stroke population.

Research is lacking on the difference grading the cognitive state of patients in dual tasks.

Therefore, identification if the performance of activity associated with the different cognitive state of patients with stroke in dual task may be meaningful to inform clinical evaluation and rehabilitation protocol.

The purpose of the present study was to investigate the performance of ability in dual task to patients with the different cognitive state post stroke.

\section{Methods}

\section{Subjects}

A convenience sample of 9 men and 3 women who had had a stroke was recruited for the study. 5 individuals had a left hemispheric stroke, and 7 individuals had a right hemispheric stroke. Participants were recruited from the Bobath Memorial Hospital. Stroke survivors were included if they met the following criteria: (1) at least 6 months after stroke as diagnosed by a medical physician, (2) ability to walk continuously for 10 meters independently without walking aid, (3) ability to hold a glass full of water with the non-affected hand, and (4) no aphasia or dysarthria interfering with verbal tasks.

Participants were excluded if they had more than one stroke, other neurologic disorders (e.g., Parkinson's disease and traumatic brain injury), and visual field defects. Cognitive status was examined using the Mini-Mental State Examination (MMSE). The study was approved by the institutional ethics committee, and participants signed written informed consent prior to participation.

\section{Study protocol}

The purpose and procedures were fully explained, and informed consents were obtained from the subjects. Each subject was evaluated individually and tested following a standard protocol.

According to the MMSE total scores, 12 patients were divided into two groups. First group was consisted of cognitively impaired patients $(n=5, M M S E<23)$. Cognitively normal patients $(n=7$, MMSE $>24)$ [17] were included in the second group.

For each subject the gait performances on Time Up and Go (TUG) test and 6 minute walk test (6MWT) were measured in three conditions: (1) preferred walking (single task); (2) for dual motor task, walking carrying a tray with glasses (tray-carrying task); (3) for dual cognitive task, walking with listening to an auditory verbal stimulus.

The instructions for each test condition were as follows: (1) "Walk with your comfortable speed right to the end of the walkway."; (2) "Walk with your comfortable speed right to the end of the walkway carrying this tray and glasses in front of you with both hands." For the tray-carrying task subjects have to keep the glasses on top of the tray without any dropping anything or else that would be considered as a fail.; (3) Walk with listening to the auditory verbal stimulus and responded by saying 'yes' when they were instructed not to the word 'red' and 'no' to the word 'blue'. If the word is neither 'red' nor 'blue', they do not response. The TUG test at each condition was repeated three successful times and the means were used for data analysis. For the 6MWT, participants were tested one time on each condition.

For the verbal stimulus, a therapist installed Recording application (Easy Voice Recorder Pro 2013) on two auditory recording devices (GALAXT NOTE $\Pi$, Samsung, Gumi, Korea, 2013) (Figure 1). The therapist recorded the words for the auditory verbal stimulus and a subject heard his voice through an earset device Bluetooth Earset (Iriver, Dongguang city, Guangdong province, China, 2013). The 


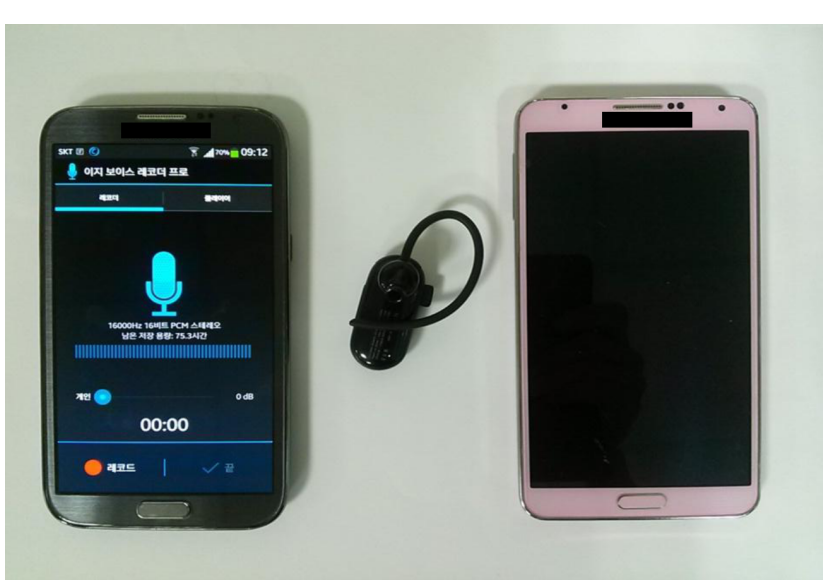

Figure 1. The auditory recording devices.

frequency range of the earset device is between 2.4-2.48 $\mathrm{GHz}$, range $10 \mathrm{~m}$, and size $42.3 \times 19.3 \times 11.6 \mathrm{~mm}$. Patients wore the earset on his/her uninvolved side (Figure 2).

\section{Procedures}

Two researchers participated in this study. One walked just behind the participant to ensure safety and the other counted error during dual tasks. Subjects took a break after each trial. We gave standard instructions to the subjects i.e. to start walking after hearing 'Ready, go', and to keep walking until asked to stop.

To reduce learning effects, participants were given only one untimed practice trial as required on both single and dual-task conditions to familiarize them with the procedure. The order of the trials was randomized and while performing dual-tasking trials there was no instruction to prioritize gait or cognitive task.

For both motor and cognitive tasks, the researcher used a stop watch to ensure accurate timing of tasks and also made a note of any inaccurate responses during the cognitive task. To measure the distance walked during 6 minutes, a participant was requested to walk at his/ her comfortable pace and to concentrate on both walking.

\section{Timed Up and Go test}

\section{Single task TUG test}

During a single task condition, Participants only performed the TUG test only (without a secondary task). A standard armchair was used, and a cone was placed at the 3-meter mark of the walking path. Participants sat comfortably with their back against the chair and the researcher gave the instruction as follows. "When I say "Go," please stand

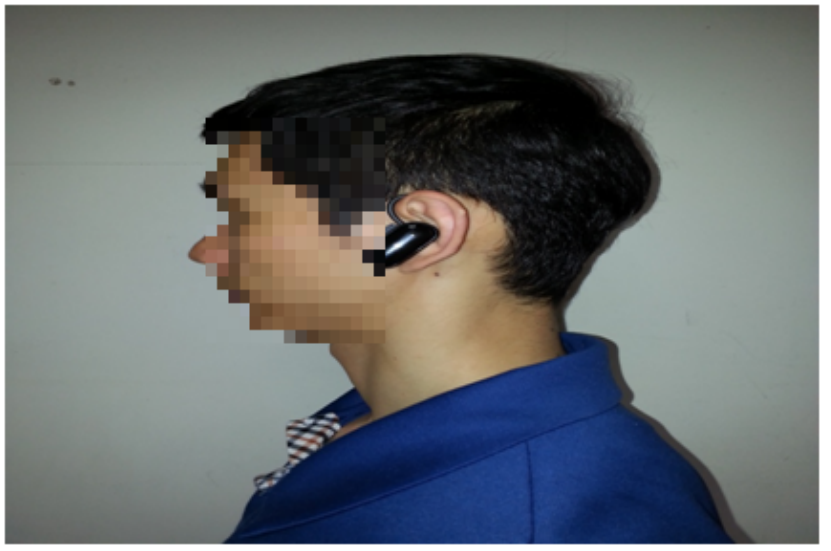

Figure 2. The auditory verbal stimulation earset device.

up from the chair and walk to the cone; turn to your right/left after you pass the cone, walk back, and sit down on the chair; please walk at your comfortable speed." We asked the participants to turn towards the paretic side for 3 trials.

\section{Dual motor task TUG test}

For the dual motor task condition, participants sat comfortably with their back against the chair while holding a glass half of water with their non-affected hand [18]. When the researcher said "Go," they stood up from the chair, walked 3 meters with a comfortable peed, turned $180^{\circ}$ towards the paretic side, walked 3 meters back to the chair, and sat down. This was repeated for 3 trials.

\section{Dual cognitive task TUG test}

Participants sat comfortably with their back against the chair. The participants walked while listening for the auditory verbal stimulus through a earset and responded by saying 'yes' when they heard the word 'red' and 'no' to the word 'blue'. If the word is neither 'red' nor 'blue', they are not to respond. When they heard "Go", they stood up from the chair, walked 3 meters with a comfortable speed, turned $180^{\circ}$ towards the paretic side, walked 3 meters back to the chair, and sat down. This was repeated for 3 times.

Two researchers followed behind a participant; one measured total distance traveled $(\mathrm{m})$ and the other recorded the number of error.

\section{6-minute walk test}

\section{Single task 6MWT}

During a single task condition, Participants only performed the 6MWT only (without a secondary task). The 
6MW was administered as a measure of walking performance. Participants were instructed to walk as fast and as far as possible in a single corridor with $180^{\circ}$ turns for 6 minutes.

\section{Dual motor task 6MWT}

For the dual motor task condition, when the researcher said "Go," participants walked in a single corridor while holding a glass half of water with their nonaffected hand [18]. Participants were instructed to walk as fast and as far as possible in a single corridor with $180^{\circ}$ turns for 6 minutes. Two researchers followed behind a participant with measuring total distance traveled $(\mathrm{m})$.

\section{Dual cognitive task 6MWT}

When the researcher said "Go," participants walked in a single corridor. The participants walked while listening for hearing the auditory verbal stimulus through a earset and responded by saying 'yes ' when they heard the word 'red' and 'no' to the word 'blue'. If the word is neither 'red' nor 'blue', they are not to respond. Two researchers followed behind a participant; one measured total distance traveled $(\mathrm{m})$ and the other recorded the number of error.

\section{Data analysis}

PASW Statistics ver. 18.0 (IBM Co., Armonk, NY, USA), was used in performance of all statistical analyses. The dependent variables were dynamic balance test and gait function. General characteristics of the subjects and variables followed a normal distribution. The independent t-test was used for analysis of between groups of dependent variables. Results were considered significant at $p<0.05$.

\section{Results}

The general characteristics of ten subjects with stroke who fulfilled the study inclusion criteria are summarized in Table 1. No significant differences were evident among the three groups in terms of baseline values. The CI group walked slower than the cognitive normal $(\mathrm{CN})$ group at the three conditions on the TUG test. However, there was no significant difference between the two groups to each condition. A significant effect of dual tasking was found only in error of verbal cognitive task condition on the TUG test. On the 6MWT, the participants in CI walked a shorter distance rather than the $\mathrm{CN}$ group. There were significant differences between the two groups not only at all conditions but also in the error of verbal cognitive task condition as well in Table 2.

For the TUG test, there was no significant difference between CI group and CN group to the mean of the TUG test. Mean of the CI group was 41.89 seconds and that of the $\mathrm{CN}$ group was 19.31 seconds.

In the condition of carrying a tray with a glass, there was no significant difference between the CI group and the $\mathrm{CN}$ group to the mean of the TUG test. The mean of the CI group was 49.98 seconds and 21.38 seconds for the $\mathrm{CN}$ group. For the dual cognitive task, in the condition of walking with listening for the auditory verbal stimulus, there was no significant difference between the CI group and the $\mathrm{CN}$ group. The mean of the CI group was 58.59 seconds and $21.21 \mathrm{sec}-$ onds for the CN group. The mean of error on the TUG test

Table 1. Characteristics of the subjects

$(\mathrm{N}=12)$

\begin{tabular}{|c|c|c|c|c|}
\hline & CI group $(n=5)$ & $\mathrm{CN}$ group $(\mathrm{n}=7)$ & $X^{2} / t$ & $p$ \\
\hline Gender (\%) & & & 0.114 & 0.735 \\
\hline Male & $4(80.00)$ & $5(70.00)$ & & \\
\hline Female & $1(20.00)$ & $2(30.00)$ & & \\
\hline Age (y) & $51.00(12.98)$ & $49.14(11.60)$ & -0.081 & 0.935 \\
\hline Height $(\mathrm{cm})$ & $169.60(6.54)$ & $165.71(7.59)$ & -0.492 & 0.623 \\
\hline Weight $(\mathrm{kg})$ & $65.18(5.76)$ & $69.76(11.03)$ & -0.895 & 0.371 \\
\hline Time since stroke $(\mathrm{mo})$ & $9.20(1.48)$ & $10.71(5.47)$ & -0.334 & 0.739 \\
\hline Side of lesion $(\%)$ & & & 0.010 & 0.921 \\
\hline Right & $3(60.00)$ & $4(54.13)$ & & \\
\hline Left & $2(40.00)$ & $3(42.87)$ & & \\
\hline Type of stroke (\%) & & & 0.010 & 0.921 \\
\hline Infarction & $2(40.00)$ & $3(42.87)$ & & \\
\hline Hemorrhage & $3(60.00)$ & $4(57.13)$ & & \\
\hline
\end{tabular}

Values are presented as $\mathrm{n}(\%)$ or mean (SD).

CI: cognitive Impairment group and below more than 23 score, CN: normal cognitive group and above more than 24 score. 
Table 2. Comparison of balance and gait measures within groups and between group $(\mathrm{N}=12)$

\begin{tabular}{|c|c|c|c|c|c|c|c|c|c|}
\hline & \multirow[b]{2}{*}{$\begin{array}{l}\text { CI group } \\
\quad(n=5)\end{array}$} & \multirow[b]{2}{*}{$\begin{array}{l}\text { CN group } \\
\quad(n=7)\end{array}$} & \multirow[b]{2}{*}{$t(p)$} & \multicolumn{3}{|c|}{ CI group $(n=5)$} & \multicolumn{3}{|c|}{$\mathrm{CN}$ group $(\mathrm{n}=7)$} \\
\hline & & & & $\begin{array}{l}\text { Control } \\
\text { vs. } \\
\text { motor }\end{array}$ & $\begin{array}{c}\text { Control } \\
\text { vs. } \\
\text { cognition }\end{array}$ & $\begin{array}{c}\text { Motor } \\
\text { vs. } \\
\text { cognition }\end{array}$ & $\begin{array}{c}\text { Control } \\
\text { vs. } \\
\text { motor }\end{array}$ & $\begin{array}{l}\text { Control } \\
\text { vs. } \\
\text { cognition }\end{array}$ & $\begin{array}{c}\text { Motor } \\
\text { vs. } \\
\text { cognition }\end{array}$ \\
\hline TUG test (sec) & $41.89(21.29)$ & $19.31(4.53)$ & 2.334 & -1.483 & -1.483 & -1.214 & $-2.366^{*}$ & $-2.028^{*}$ & -0.338 \\
\hline TUG test (cup) & $49.98(28.26)$ & $21.38(4.59)$ & 2.242 & & & & & & \\
\hline TUG test (yes/no) & $58.59(38.27)$ & $21.21(5.46)$ & 2.168 & & & & & & \\
\hline TUG test yes/no (error) & $11.70(8.26)$ & $0.39(0.49)$ & $3.060^{*}$ & & & & & & \\
\hline $6 \mathrm{MWT}(\mathrm{m})$ & $115.97(66.17)$ & $212.03(56.99)$ & $-2.679^{*}$ & $-2.023^{*}$ & -1.753 & -0.405 & $-2.366^{*}$ & -1.352 & -1.521 \\
\hline 6MWT (cup) & $101.57(59.65)$ & $185.87(57.24)$ & $-2.473^{*}$ & & & & & & \\
\hline $6 \mathrm{MWT}($ yes/no) & $99.68(48.19)$ & $198.93(58.26)$ & $-3.112^{*}$ & & & & & & \\
\hline 6MWT yes/no (error) & $66.40(32.32)$ & $1.86(2.12)$ & $4.458^{*}$ & & & & & & \\
\hline
\end{tabular}

Values are presented as mean (SD).

CI: cognitive impairment, CN: cognitive normal, TUG test: Timed Up and Go test (cup: dual motor task, yes/no: dual cognitive task), 6MWT: 6 minute walk test.

${ }_{p}^{*}<0.05$.

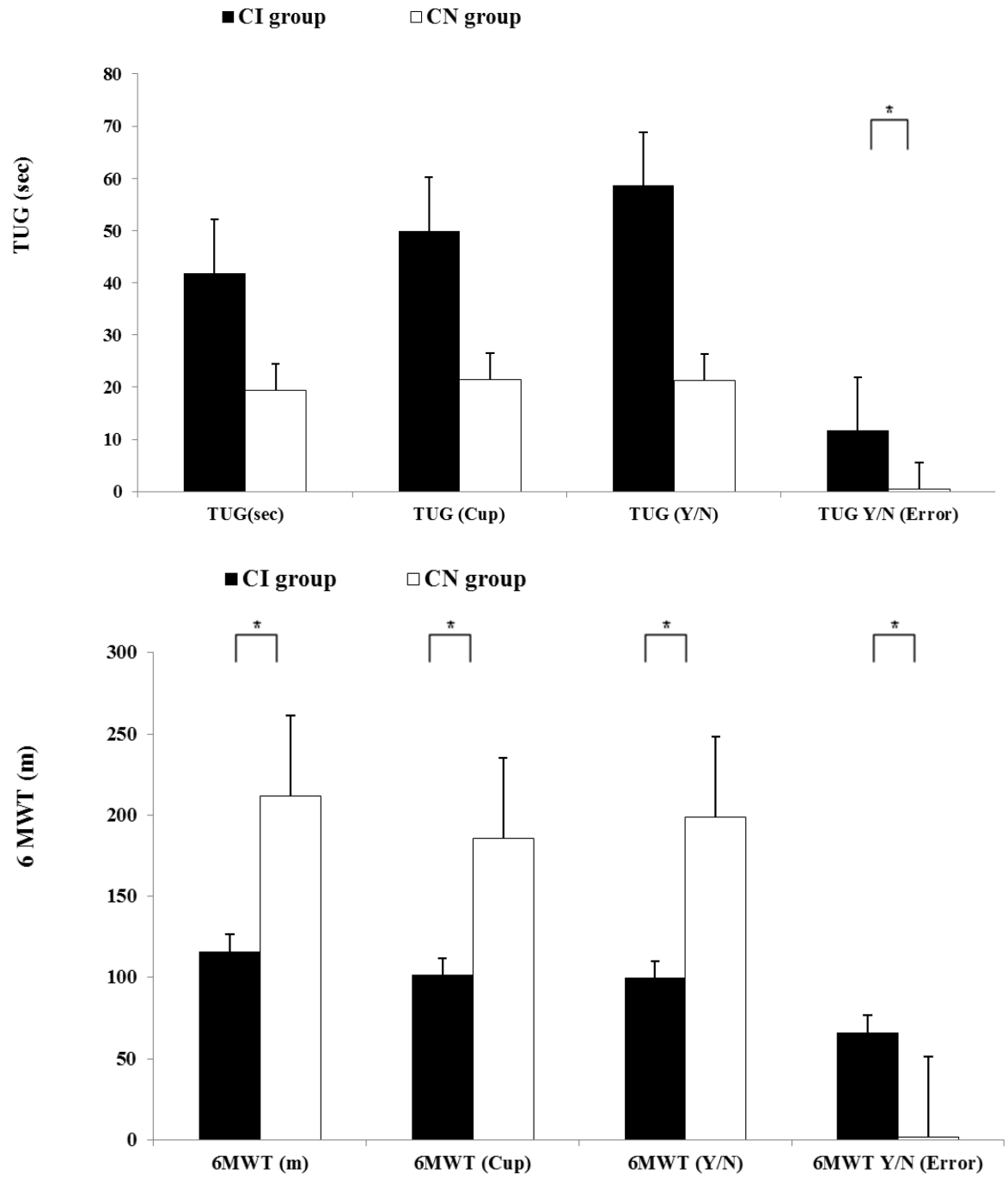

Figure 3. Differences of balance and gait parameter in cognitive impairment (CI) group and cognitive normal $(\mathrm{CN})$ group. *Significant difference between $\mathrm{CI}$ group and $\mathrm{CN}$ group $(p<0.05)$. TUG test: Timed Up and Go test, 6MWT: 6 minute walk test, Y/N: yes/no (cup: dual motor task, yes/no: dual cognitive task). 
during dual cognitive task was 11.70 times in the CI group and 0.39 times in the $\mathrm{CN}$ group. There was a significant difference between groups with a $p$-value of 0.037 . For the 6MWT, there was a significant difference between the CI group and the $\mathrm{CN}$ group. The mean of the $\mathrm{CI}$ group was $115.97 \mathrm{~m}$, and $212.03 \mathrm{~m}$ for the $\mathrm{CN}$ group $(p=0.022$ ). In the condition of carrying a tray with a glass, there was a significant difference between the $\mathrm{CI}$ group and the $\mathrm{CN}$ group. The mean of the CI group was $101.57 \mathrm{~m}$, and $185.87 \mathrm{~m}$ for the $\mathrm{CN}$ group ( $p=0.033$ ). For dual cognitive task, in the condition of walking with hearing the auditory verbal stimulus, there was a significant difference between the CI group and the $\mathrm{CN}$ group $(p=0.011$ ). The mean of the CI group was $99.68 \mathrm{~m}$ and that of $\mathrm{CN}$ group was $198.93 \mathrm{~m}$. The mean of error on the 6MWT during dual cognitive task was 66.40 times in CI group and 1.86 times in $\mathrm{CN}$ group. There was a significant difference between groups $(p=0.011)$ (Figure 3).

\section{Discussion}

Standing balance might influence ambulation; however, other factors are necessary for building community ambulation. It is concluded that combining the walking ability with other factors such as cognitive and behavioral functions is necessary to achieve the community ambulation [15].

In this study, the factors that may affect community ambulation like balance, motor functions, and endurance, walking speed were evaluated with the TUG test and the 6MWT. In addition, this study has demonstrated the effect of various dual tasks between impaired cognition and normal cognition in patients with stroke.

Paker et al. [19] reported functional follow-up parameters were significant improved in both the cognitively impaired and normal groups. However, it did not show statistically significant difference statistically between the groups. The community ambulation rate was higher in the cognitively normal group. On short distance such as TUG test in this study, although there was no significant difference between the $\mathrm{CI}$ group and the $\mathrm{CN}$ group in three conditions; only walking, dual motor task (walking with carrying tray), and dual cognitive task (walking with verbal response), participants demonstrated deterioration to complete TUG test including longer time both $\mathrm{CI}$ group and $\mathrm{CN}$ group. This finding corresponds that Manaf et al. [20] found the time taken to complete the TUG test across different attentional conditions (dual motor and dual-cognitive) in patients with stroke.

However, on the 6MWT in the conditions, there was significant difference between both groups. The 6MWT is prolonged walking and is used to measure endurance ability [21]. Especially, participants in the CI group demonstrated a higher number of error when they were required to perform dual cognitive task on the 6MWT than that of the $\mathrm{CN}$ group.

Patient characteristics, provider perceptions, practice guidelines, and available treatments may all play a role in shaping the impact of CI on stroke rehabilitation outcomes [22]. For instance, a cognitively impaired patient may not be able to provided the same level of rehabilitation because of his/her impairment [23]. Therefore, cognitively impaired patients may not be able to benefit fully from rehabilitation because of their impairment [24]. Research suggests that this may occur in patients with a sensory deficit (contralateral neglect), deficits of attention, or impaired comprehension and learning $[25,26]$. Previous research has suggested that mathematical subtractions (which relies more on working memory) generate a greater cognitive load than verbal fluency (which relies more on semantic memory) in frail older adults [27]. Others have suggested real-time feedback on balance and gait performance of chronic hemiparetic stroke survivors. For example, as Chung et al. [28] reported that core stabilization exercise with real-time feedback improved balance and gait function in patients with stroke patients.

Even though the CI group showed difference of mean intra-group, there was no significant difference with three conditions on TUG test. It means that the participants with CI were not affected to type of dual task on the TUG test.

While comparing the error of the TUG test between the CI and the $\mathrm{CN}$ groups, the $\mathrm{CI}$ group made an average of $11.7 \mathrm{er}-$ rors composed 0.39 errors made by the $\mathrm{CN}$ groups. The rate of error on the 6MWT was 66.40 times in the CI group and 1.86 times in the CA group. To consider the results, participants in the CI group did not pay attention to the therapist's instruction and frequently made mistakes not to relate with distance. For the CI group, it is better to use a cognitive task rather than a motor task on the TUG test because walking speed decreased significantly. Kim and Lee [29] reported the action observation training that is a cognitive intervention method was significant improvement in the TUG test on stroke survivors. CI side should be treated due to decrease walking speed. Shin and Lee [30] reported that the average decrease in the time required during TUG test with 
additional weight was 14.57 seconds, a 55\% improvement. The additional weight consists of two devices (weights and jacket) and two different weights $(0.1 \mathrm{~kg}$ and $0.5 \mathrm{~kg})$ were used during gait training. Chung and Lee [31] used treadmill gait training to investigate TUG test for patients with stroke and got a result of decreased TUG score. Noha et al. [32] got a result that TUG showed significant improvement with use of the elastic band orthosis.

When considering the mean of the 6MWT, long distance, the $\mathrm{CI}$ groups was affected from cognitive tasks rather than motor tasks, but the $\mathrm{CN}$ group did affect from motor.

This study has several limitations. First, one is the low number of patients as a sample size. Second, although participants were randomly assigned, we did not analyze their long-term outcomes. This study mainly focuses on cross-sectional observation. Therefore, we suggest future study with use of a different study design such as randomized control trial. It may increase potential clinical benefits. Lee and Choi [33] reviewed the effects of dual-task training for stroke survivors. They introduced that Yang et al. [34] reported that the walking variables between socially independent group and limited group after stroke showed no big difference. While doing dual-task rather than single task, walking speed of all group were decreased.

To consider the results of different distances such as the TUG test and the 6MWT, we may suggest that exercises in long distance would be more effective for patients with CI. This would improve the patient's endurance with a cognitive problem.

\section{Conflict of Interest}

The authors declared no potential conflicts of interest with respect to the authorship and/or publication of this article.

\section{References}

1. Hausdorff JM, Yogev G. Cognitive function may be important for fall injury prevention trials. J Am Geriatr So 2006;54:865; author reply 865-6.

2. Hausdorff JM, Yogev G, Springer S, Simon ES, Giladi N. Walking is more like catching than tapping: gait in the elderly as a complex cognitive task. Exp Brain Res 2005;164:541-8.

3. Plummer-D'Amato P, Altmann LJ, Saracino D, Fox E, Behrman $\mathrm{AL}$, Marsiske M. Interactions between cognitive tasks and gait after stroke: a dual task study. Gait Posture 2008;27:683-8.

4. Hauer K, Pfisterer M, Weber C, Wezler N, Kliegel M, Oster P. Cognitive impairment decreases postural control during dual tasks in geriatric patients with a history of severe falls. J Am Geriatr Soc 2003;51:1638-44.

5. Yogev-Seligmann G, Rotem-Galili Y, Mirelman A, Dickstein R, Giladi N, Hausdorff JM. How does explicit prioritization alter walking during dual-task performance? Effects of age and sex on gait speed and variability. Phys Ther 2010;90:177-86.

6. Hyndman D, Ashburn A, Yardley L, Stack E. Interference between balance, gait and cognitive task performance among people with stroke living in the community. Disabil Rehabil 2006;28:849-56

7. Yang YR, Chen YC, Lee CS, Cheng SJ, Wang RY. Dual-task-related gait changes in individuals with stroke. Gait Posture 2007;25:185-90.

8. Canning CG, Ada L, Paul SS. Is automaticity of walking regained after stroke? Disabil Rehabil 2006;28:97-102.

9. Bowen A, Wenman R, Mickelborough J, Foster J, Hill E, Tallis R. Dual-task effects of talking while walking on velocity and balance following a stroke. Age Ageing 2001;30:319-23.

10. Cockburn J, Haggard P, Cock J, Fordham C. Changing patterns of cognitive-motor interference (CMI) over time during recovery from stroke. Clin Rehabil 2003;17:167-73.

11. Naço D, Dobi D, Zekja I, Mijo S, Kapisyzi M, Kruja J. Factors influencing mini-mental state (MMSE) score in stroke patients. Med Arch 2013;67:171-3.

12. Diamond PT, Felsenthal G, Macciocchi SN, Butler DH, Lally-Cassady D. Effect of cognitive impairment on rehabilitation outcome. Am J Phys Med Rehabil 1996;75:40-3.

13. Stephens S, Kenny RA, Rowan E, Kalaria RN, Bradbury M, Pearce R, et al. Association between mild vascular cognitive impairment and impaired activities of daily living in older stroke survivors without dementia. J Am Geriatr Soc 2005;53:103-7.

14. Tatemichi TK, Desmond DW, Stern Y, Paik M, Sano M, Bagiella E. Cognitive impairment after stroke: frequency, patterns, and relationship to functional abilities. J Neurol Neurosurg Psychiatry 1994;57:202-7.

15. Lord SE, Rochester L. Measurement of community ambulation after stroke: current status and future developments. Stroke 2005;36:1457-61.

16. Regnaux JP, David D, Daniel O, Smail DB, Combeaud M, Bussel B. Evidence for cognitive processes involved in the control of steady state of walking in healthy subjects and after cerebral damage. Neurorehabil Neural Repair 2005;19:125-32.

17. Folstein MF, Folstein SE, McHugh PR. "Mini-mental state". A practical method for grading the cognitive state of patients for the clinician. J Psychiatr Res 1975;12:189-98.

18. Shumway-Cook A, Brauer S, Woollacott M. Predicting the probability for falls in community-dwelling older adults using the Timed Up \& Go Test. Phys Ther 2000;80:896-903.

19. Paker N, Buğdaycı D, Tekdöş D, Kaya B, Dere C. Impact of cognitive impairment on functional outcome in stroke. Stroke Res Treat 2010;2010.

20. Manaf H, Justine M, Omar M. Functional balance and motor impairment correlations with gait parameters during Timed Up and Go test across three attentional loading conditions in stroke survivors. Stroke Res Treat 2014;2014:439304.

21. Iosa M, Morone G, Fusco A, Pratesi L, Bragoni M, Coiro P, et al. Effects of walking endurance reduction on gait stability in patients with stroke. Stroke Res Treat 2012;2012:810415. 
22. Zinn S, Dudley TK, Bosworth HB, Hoenig HM, Duncan PW, Horner RD. The effect of poststroke cognitive impairment on rehabilitation process and functional outcome. Arch Phys Med Rehabil 2004;85:1084-90.

23. Gray DS. Slow-to-recover severe traumatic brain injury: a review of outcomes and rehabilitation effectiveness. Brain Inj 2000;14:1003-14.

24. Robertson IH, Ridgeway V, Greenfield E, Parr A. Motor recovery after stroke depends on intact sustained attention: a 2-year follow-up study. Neuropsychology 1997;11:290-5.

25. Mysiw WJ, Beegan JG, Gatens PF. Prospective cognitive assessment of stroke patients before inpatient rehabilitation. The relationship of the Neurobehavioral Cognitive Status Examination to functional improvement. Am J Phys Med Rehabil 1989;68: 168-71.

26. Rose L, Bakal DA, Fung TS, Farn P, Weaver LE. Tactile extinction and functional status after stroke. A preliminary investigation. Stroke 1994;25:1973-6.

27. Beauchet O, Dubost V, Gonthier R, Kressig RW. Dual-task-related gait changes in transitionally frail older adults: the type of the walking-associated cognitive task matters. Gerontology 2005;51:48-52.
28. Chung E, Lee BH, Hwang S. Core stabilization exercise with real-time feedback for chronic hemiparetic stroke: a pilot randomized controlled trials. Restor Neurol Neurosci 2014;32:313-21.

29. Kim JH, Lee BH. Action observation training for functional activities after stroke: a pilot randomized controlled trial. NeuroRehabilitation 2013;33:565-74.

30. Shin SH, Lee MY. Effect of gait training with additional weight on balance and gait in stroke patients. Phys Ther Rehabil Sci 2014;1:55-62.

31. Chung EJ, Lee BH. The effects of treadmill training on dynamic balance and gait function in stroke patients: a pilot randomized controlled trial. Phys Ther Rehabil Sci 2013;1:39-43.

32. Noha D, Lee SG, Yang YJ. Effects of elastic band orthosis (aider) on balance and gait in chronic stroke patients. Phys Ther Rehabil Sci 2013;2:81-6.

33. Lee GC, Choi WJ. The effects of dual-task training on ambulatory abilities of stroke patients: Review of the latest trend. Phys Ther Rehabil Sci 2012;1:1-5.

34. Yang YR, Chen YC, Lee CS, Cheng SJ, Wang RY. Dual-task-related gait changes in individuals with stroke. Gait Posture 2007; 25:185-90. 